\title{
Awareness of national malaria management guidelines among house officers in Khartoum state teaching hospitals. Sudan 2018
}

Mohammed Altyb Alshykh Aboshanab ( $\sim$ Bonish3434@gmail.com )

Research article

Keywords: Malaria, management, guidelines, house officers

Posted Date: December 12th, 2019

DOI: https://doi.org/10.21203/rs.2.18883/v1

License: (c) (i) This work is licensed under a Creative Commons Attribution 4.0 International License.

Read Full License 


\section{Abstract}

Background: Malaria is a protozoan disease which can lead to serious complications if not treated early and correctly. The aim of this study to assess the knowledge about malaria management guidelines among house officers.

Methods: this is the cross sectional observational study conducted at 6 of Khartoum teaching hospitals

Results: The study showed that among the 115 participants evaluated there were $70.4 \%$ females and $29.6 \%$ were males. $94.8 \%$ of participants knew there is malaria management guidelines and $5.2 \%$ didn't knew about presence of this guidelines, $58.3 \%$ have some information and $10.4 \%$ just hear about this guidelines. $89.6 \%$ knew the management of simple malaria is an outpatient management. $65.2 \%$ of the participants were aware about where to manage the cases of complicated malaria (inpatient or in Intensive Care Unit). $27.8 \%$ of the participants were aware about the management of simple malaria in the second and third trimester.

Conclusion: Different levels of awareness about malaria management guidelines as whole: $4.3 \%$ had an overall poor level of awareness, and $74.8 \%$ had an overall average level of awareness and $20.9 \%$ had an overall good level of awareness.

\section{Introduction}

The aims of medical guideline are guiding decisions and criteria in treatment of specific areas of healthcare regarding diagnosis, management. Such guidelines have been use during the entire history of medicine for thousands of years [1]. It recommend specific care for people with specific conditions and determine how healthcare professionals should deal with it [2].

Malaria is a protozoan disease transmitted by the bite of infected Anopheles mosquitoes by plasmodium parasites. The species of plasmodium [3] are P. falciparum, P. vivax, P. ovale, and P. malariae and almost all deaths are caused by falciparum malaria [4]. The clinical features are headache, malaise, hepatosplenomegaly, anemia and vomiting. Jaundice is common due to hemolysis. The clinical features of malaria are non-specific and the diagnosis must be suspected in anyone returning from an endemic area who has features of infection. The risk of severe malaria. P. vivax and P. ovale increase with previous splenectomy [5]. All patients with suspected malaria should be treated on the basis of a confirmed diagnosis by microscopy examination or RDT testing of a blood sample. High specificity will reduce unnecessary treatment with antimalarial drugs and improve the diagnosis of other febrile illnesses in all setting[6]. The management of malaria based on the severity if it is simple malaria treated by Artemether + lumefantrine according to WHO guidelines [6]. If it is complicated malaria the patient must be hospitalized and treated with artesunate IV quinine IV [7]. During pregnancy the treatment is different according to severity and the trimester. In first trimester if it is simple malaria treated by Quinine plus clindamycin or quinine monotherapy. In second trimester treated by Artemether + lumefantrine or any one 
from ACT drugs group or artesunate plus clindamycin or quinine. If it is complicated malaria in all trimester treated by artesunate or quinine [8]

The aim of this study to assess the house officers knowledge about malaria management guidelines and this study will contribute to increase of teaching and make the junior doctors adhere to this guidelines.

\section{Methods}

This is a cross sectional observational study was conducted at 6 of Khartoum state teaching hospitals are chosen which include Khartoum Bahri teaching hospital, Ahmed Gassim teaching hospital, Haj Al Safi teaching hospital, EL ban Gadeed teaching hospital, Ibrahim Malik teaching hospital, and EL academe teaching hospital. Include 115 participants distributed at year of 2018. The Inclusion criteria are House officers whom distributed during the mentioned period and completed or currently enrolled in the internal medicine shift. Excluded those who didn't complete or not currently enrolled in the internal medicine shift and those who are registered outside the time period mentioned. Those who refuse to participate. The data was collected by a structured questionnaire, self-administered using WHO 2015 as guidelines for malaria management. The data was categorized and analyzed using IBM SPSS statistics 20.

\section{Results}

The study showed that among the 115 participants evaluated there were $70.4 \%$ females and $29.6 \%$ were males. $99.1 \%$ are aged $20-29$ and only $0.9 \%$ are aged $30-39$. The most common source was medical school $54.8 \%$ and the head of the medical unit 36.5\%. (Table 1). We targeted the Khartoum teaching hospitals where $22.6 \%$ of participants was from Khartoum Bahri teaching hospital, $25.2 \%$ from Ibrahim Malik teaching hospital, 18.3\% from Ahmed Gassim, teaching hospital, 15.7\% from El academe teaching hospital, 11.3\% from Haj Alsafi teaching hospital and 7\% from El ban Gadeed teaching hospital (Table 2). $94.8 \%$ of participants knew there is malaria management guidelines and $5.2 \%$ didn't knew about presence of this guidelines, $58.3 \%$ have some information and $10.4 \%$ just hear about this guidelines (Table 3 ). $89.6 \%$ knew the management of simple malaria is an outpatient management. $71.3 \% \mathrm{knew}$ the first line of treatment (lumefantrine + artemether) and only $33 \%$ of them knew the second line for the management of simple malaria (Table 4). $65.2 \%$ of the participants were aware about where to manage the cases of complicated malaria (inpatient or in ICU) and $74.8 \%$ knew the treatment of complicated malaria (Table 5). $71.3 \%$ knew the first line of management of malaria in the first trimester (oral quinine). Only $27.8 \%$ of the participants were aware about the management of simple malaria in the second and third trimester. $83.5 \%$ knew the management of complicated malaria during pregnancy. $36.5 \%$ knew the treatment of malaria caused by plasmodium vivax and ovale (Table 6)

The level of awareness of the participants; $4.3 \%$ had an overall poor level of awareness "answered less than one third of the questions correctly", and $74.8 \%$ had an overall average level of awareness "answered more than one third of the questions correctly" and $20.9 \%$ had an overall good level of awareness "answered more than two thirds of the questions correctly" (Table 7) 


\section{Discussion}

The implementation of a national drug policy faces several constraints, such as the logistics of distribution, the large number and variety of people and institutions involved and the rising cost of

treatment. Appropriate planning is therefore essential for successful implementation. In this study, some constraints and problems were highlighted which reflect on the implementation of the protocol for national malaria control [9].

The study showed that among the 115 participants evaluated there were 70.4 females, 29.6 males and almost of all of them were aged 20-29 years and the levels of awareness about malaria management guidelines as whole was as follows: $4.3 \%$ had an overall poor level of awareness, $74.8 \%$ had an overall average level of awareness and $20.9 \%$ had an overall good level of awareness. Which means $95.3 \%$ have an accepted level of awareness about malaria management guidelines in contrast to another study which the level of awareness of house officers about the malaria management guidelines was only $37.9 \%$ that because the Khartoum state contain a lot of hospitals and it contain a lot of house officers [9].

The weakest point in the awareness was the second line of treatment of simple malaria, management of malaria during second and third pregnancy and management of malaria caused by vivax and ovale species of malaria most probably due to the poor exposure of fresh doctors like the house officers to the fairly uncommon situations. Aware about the management of simple malaria in the second and third trimester.

The limitation of this study are small sample size due to refusal of the Sudan medical council to provide us with the statistics about the registered house officers we was select 115 house officers as sample size which we only think it will be representative with a total of which was distributed among the 6 hospitals.

\section{Conclusion}

Different levels of awareness about malaria management guidelines as whole: $4.3 \%$ had an overall poor level of awareness, and $74.8 \%$ had an overall average level of awareness and $20.9 \%$ had an overall good level of awareness.

\section{Declarations}

\section{Ethical consideration:}

The ethics committee at each hospital was notified and permission taken, each participant was asked for verbal consent before participating and all conflicts was discussed.

\section{Availability of data and materials:}

The data generated and analyzed during the current study are not publicly available because this paper not sent to any perreview. 
Competing interest:

No one of authors has a conflict of interest

Funding:

This study was not received any financial supports.

\section{Authors' contribution:}

Mohammed Altyb Alshykh Aboshanab, Mohammed Altayeb AbdAlhafeez Mohammed, Mohamed Elzubair Mohamed Ataelmanan, Mohammed Alsheekh Alaqbash and Muhsin Mohammed Elhadi Mohammed: All of those authors contribute in data collection, data analysis and writing.

Nagla Abdelrahim Mohamed Ahmed: Supervised all steps of completion of this paper

\section{Acknowledgements}

Thanks to Dr. Nagla Abdelrahim Mohamed Ahmed Community Medicine Department, faculty of medicine- university of AL Zaiem Al azhari University for her support and supervision of us in our study and to all whom helped us to accomplish this works and to our families and friends for their continued support.

\section{Abbreviations}

ACT: Artesunate combined therapy

AL: Artemether-lumefantrine

AS: Artesunate

DHA: Dihydroartemisinin

EMT: Emergency medical technician

ECG: Electrocardiogram

EU: Europe

G6PD: Glucose 6 phosphate dehydrogenase

HIV: Human immunodeficiency virus

IM: Intramuscular

IV: Intravenous 
KAP: Knowledge attitude and practice

MQ: Mefloquine

NHS: National Health Service

P: Plasmodium

PPQ: Piperaquine

RBC: Red blood cells

RDT: Rapid diagnostic test

SP: Sulfadoxine - pyrimethamine

SPSS: Statistical package of social sciences

UK: United Kingdom

WHO: World health organization.

\section{References}

1. JAKO S. Burgers, R.G., Niek S. Klazinga, Marjukka Makela, Joost Zaat Towards evidence-based clinical practice: an international survey of 18 clinical guideline programs. Int J Qual Health Care, 2003. 15: p. 31-45.

2. Steven H Woolft, R.G., Allen Hutchinson, Martin Eccles and Jeremy Grimshaw, Potential benefits, limitations, and harms of clinical guidelines. 1999. 318( 7182): p. 527-530.

3. wikipedia. medical protocoles. 3 January 2018; Available from: https://en.wikipedia.org/wiki/Medical_protocol.

4. Harrison, malaria, in Harrison principles of internal medicine. 2010, The McGraw-Hill Companies, Inc. p. 1077-1078.

5. Davidson., malaria, in Davidson princeples and prctice of medicine 2014, Elsevier p. 355-357.

6. WHO, Guidelines for the treatment of malaria. 2015, WHO Library. p. 27,32-34,59,72.

7. veronique grouzard, J.R., Marianne Sutton, malaria management, in clinical guidelines diagnosis and treatment manual. 2016, medecins san forntieres. p. 141.

8. WHO, WHO Guidance for Prevention and Treatment of Malaria in Pregnancy, in Treatment of Malaria in Pregnancy. 2010. p. 2-3.

9. Yousif, M.E.A.a.M.A., Impact of the national protocol for malaria treatment on prescribing patterns in Gezira state, Sudan. Mediterranean Health Journal, 2004. 10: p. 566-57210. 


\section{Tables}

Table 1: This table shows the gender, Age of participants and the Source of the information about the malaria guidelines:

\begin{tabular}{ccc}
\hline Variables & Frequency & Percent \\
& $\mathrm{N}=115$ & \\
\hline Female & 81 & $70.4 \%$ \\
Male & 34 & $29.6 \%$ \\
Age & & \\
& & \\
20-29 years & 114 & $99.1 \%$ \\
30-39 years & 1 & $0.9 \%$ \\
Source of information & & \\
College & 63 & $54.8 \%$ \\
Workshop & 33 & $28.7 \%$ \\
Head of the medical unit & 42 & $36.5 \%$ \\
Internet & 23 & $20 \%$ \\
Colleagues & 28 & $24.3 \%$ \\
\hline
\end{tabular}

Table 2: This table shows the Khartoum teaching hospitals where the data have been collected:

\begin{tabular}{ccc}
\hline Hospital's name & Frequency & Percent \\
& $\mathrm{N}=115$ & \\
\hline AHMED GASSIM, TEACHING HOSPITAL & 21 & 18.3 \\
EL ACADEME TEACHING HOSPITAL & 18 & 15.7 \\
EL BAN GADEED TEACHING HOSPITAL & 8 & 7.0 \\
HAJ ALSAFI TEACHING HOSPITAL & 13 & 11.3 \\
IBRAHIM MALIK TEACHINGT HOSPITAL & 29 & 25.2 \\
KHARTOUM BAHRY TEACHING HOSPITAL & 26 & 22.6 \\
\hline
\end{tabular}

Table 3: This table shows the awareness about presence of malaria management guidelines: 


\begin{tabular}{ccc}
\hline Variables & Frequency & Percent \\
& $\mathrm{N}=115$ & \\
\hline NO presence of malaria guidelines & 6 & $5.2 \%$ \\
Presence of malaria guidelines & 109 & $94.8 \%$ \\
Good information about it. & 36 & $31.3 \%$ \\
Some information about it. & 67 & $58.3 \%$ \\
Just hear about it but I have no idea. & 12 & $10.4 \%$ \\
\hline
\end{tabular}

Table 4: This table shows the awareness about management of simple malaria:

\begin{tabular}{lcc}
\hline \multicolumn{1}{c}{ Variables } & Frequency (N=115) & Percent \\
\hline Outpatient management of simple malaria & 103 & 89.6 \\
Inpatient management of simple malaria & 12 & 10.4 \\
First line of treatment for simple malaria: & & \\
A. Quinine & 11 & 9.6 \\
B. lumefantrine & 12 & 10.4 \\
C. lumefantrine + artemether & 82 & 71.3 \\
D. IV artesunate. & 10 & 8.7 \\
Second line of treatment for simple malaria & & 33.0 \\
A. Dihydroartemisinin + piperaquine or Quinine & 38 & 29.6 \\
B. sulfadoxine-pyrimethamine + artesunate & 34 & 13.0 \\
C. Co-artemether & 15 & 24.3 \\
D. IV artesunate & 28 & \\
\hline
\end{tabular}

Table 5: This table shows the awareness about management of complicated malaria: 


\begin{tabular}{lll}
\hline \multicolumn{1}{c}{ Variables } & Frequency & Percent \\
& $\mathrm{N}=115$ & \\
\hline A. Outpatient & 6 & 5.2 \\
B. Inpatient. & 29 & 25.2 \\
C. ICU & 5 & 4.3 \\
D. inpatient or ICU & 75 & 65.2 \\
Treatment of complicated malaria & & \\
A. Artemether IM & 17 & 14.8 \\
B. Quinine IV or artesunate IV & 86 & 74.8 \\
C. sulfadoxine-pyrimethamine + artesunate & 3 & 2.6 \\
D. Co-artemether & 9 & 7.8 \\
\hline
\end{tabular}

Table 6: this table shows the awareness about management of malaria in pregnancy, and the management of malaria caused by P. vivax and P. ovale: 


\begin{tabular}{|c|c|c|}
\hline Variables & $\begin{array}{l}\text { Frequency } \\
\mathrm{N}=115\end{array}$ & Percent \\
\hline \multicolumn{3}{|l|}{ Treatment of simple malaria in first trimester } \\
\hline A. Artesunate & 18 & 15.7 \\
\hline B. Sulfadoxine-pyrimethamine + artesunate. & 7 & 6.1 \\
\hline C. oral Quinine & 82 & 71.3 \\
\hline D. Co-artemether. & 8 & 7.0 \\
\hline \multicolumn{3}{|l|}{ Treatment of simple malaria in second and third trimester } \\
\hline A. Artemether + lumefantrine & 15 & 13.0 \\
\hline B. Artesunate. & 17 & 14.8 \\
\hline C. Quinine. & 51 & 44.3 \\
\hline D. All of this drugs & 32 & 27.8 \\
\hline \multicolumn{3}{|l|}{ Treatment of complicated malaria in all trimester } \\
\hline A. Quinine or Artesunate & 96 & 83.5 \\
\hline B. Dihydroartemisinin + piperaquine & 6 & 5.2 \\
\hline C. Sulfadoxine-pyrimethamine + artesunate. & 6 & 5.2 \\
\hline D. Artemether + lumefantrine. & 7 & 6.1 \\
\hline \multicolumn{3}{|l|}{ Treatment of malaria caused by $P$. vivax and $P$. ovale } \\
\hline A. Artesunate IV or IM. & 4 & 3.5 \\
\hline B. Quinine. & 17 & 14.8 \\
\hline C. Primaquine. & 52 & 45.2 \\
\hline D. Artemether + lumefantrine after that Primaquine. & 42 & 36.5 \\
\hline
\end{tabular}

Table 7: This table shows level of awareness: 
Level Frequency Percent

$$
\mathrm{N}=115
$$

$\begin{array}{lll}\text { Poor level } & 5 & 4.3\end{array}$

Average level 86

74.8

Good level $\quad 24 \quad 20.9$ 